\title{
Society for Pediatric Research Presidential Address 2001: Pediatric Research in the Era of Genomics
}

\author{
ORA HIRSCH PESCOVITZ \\ Section of Pediatric Endocrinology and Diabetology, Department of Pediatrics, Riley Children's Hospital, \\ and the Office of the Dean, Indiana University School of Medicine.
}

For the past year, it has been my privilege to serve as the president of the Society for Pediatric Research, and I am delighted to be here today to give the SPR presidential address. Like others before me, I have been stricken by the PAS: the Presidential Address syndrome - a syndrome that was first described by Dr. Melvin Grumbach, when he was president of the APS (1). This is a syndrome that has plagued most PAS presidents before me. The symptoms begin approximately 5 minutes after the results of the election are announced and the vice-president is hit with the realization that the presidential address is only 24 months away!! The syndrome is characterized by feelings of inadequacy, often coupled with systemic symptoms including headache and gastrointestinal distress. This debilitating condition is cured remarkably within moments of the completion of the president's term.

I have chosen to address the topic of Pediatric Research in the Era of Genomics. This is an exciting time for research in general, and for pediatric research in particular. There is strong bipartisan support for increasing the federal investment in research, and never before has there been more National Institutes of Health funding for research (2). The federal government has supported an impressive doubling of the $\mathrm{Na}$ tional Institutes of Health budget from \$13.7 billion in 1998 to an expected \$27.4 billion in 2003 (Fig. 1). Although the current administration has not proposed generous support specifically for children's hospitals, support for research, including pediatric research, is at an all-time high.

In addition to federal support, corporate support also increased dramatically in the 1990s (3). In fact, industry support for research has surpassed federal funding (Fig. 2). So, in this era of outstanding research funding, what is the status of pediatric research?

In the year 2000, departments of pediatrics received approximately $3.2 \%$ of the total National Institutes of Health dollars awarded for research (data supplied by Dr. Duane Alexander, NICHD, National Institutes of Health; Fig. 3). This was similar to the percent of National Institutes of Health dollars awarded to departments of pediatrics in the early 1990s. Thus, the data indicate that pediatric departments were able to sustain increases in research funding comparable to the national average

\footnotetext{
August 7, 2001; August 14, 2001.

Correspondence and reprint requests: Ora Hirsch Pescovitz, MD, Riley 5960, 702 Barnhill Drive, Indianapolis, Indiana 46032; e-mail: opescovi@iupui.edu

DOI: 10.1023/01.PDR.0000015611.76067.66
}

during the past decade. Most National Institutes of Health dollars awarded to pediatric investigators came from NHLBI, NICHD, NIAID, NCI, and the NIDDK. These institutes spent between $10 \%$ and $24 \%$ of their research dollars to support research done in pediatric departments (Fig. 4).

In the United States, there are 125 departments of pediatrics in medical schools and 52 freestanding children's hospitals. Some very important pediatric research is performed in the outpatient, private clinical setting such as through the PROS (Pediatric Research in the Office Setting) network. However, for the purposes of this analysis, I have assumed that most pediatric research takes place in either pediatric departments or freestanding children's hospitals.

Although pediatric research funding has kept pace with the overall increases in National Institutes of Health funding, it is notable that most pediatric institutions conduct little research (Fig. 5). Using 1999 data extracted from the NACHRI database (4), and assuming that there are 177 pediatric institutions (125 departments and 52 freestanding children's hospitals), 97 of them had almost no National Institutes of Health funding, 46 had less than $\$ 5$ million in annual National Institutes of Health funding for research, 19 institutions had between $\$ 5$ and 10 million, 10 institutions received between $\$ 10$ and 20 million and only 5 institutions had more than \$20 million.

Who does pediatric research? There are few statistics on the number of academic general pediatricians, behavioral and developmental pediatricians, and Ph.D. scientists who are actively engaged in pediatric research. There are data from the American Board of Pediatrics regarding pediatric subspecialists (5). Foreign medical graduates account for $40 \%$ of pediatric subspecialists and there are approximately equal numbers of men and women in pediatric subspecialties today. Since not all subspecialists are actively engaged in research, I reviewed additional data from the American Board of Pediatrics to determine the percentage of pediatricians in each subspecialty that by self-report practice in an academic medical setting (Fig. 6 ). For example, $76 \%$ of emergency room pediatricians work in academic medical settings. In contrast, only $36 \%$ of neonatologists work in the academic environment. The total number of subspecialists in each subspecialty who report working in full-time academic medicine can be reviewed in Figure 7.

In total, there are only 6,765 pediatric subspecialists working in academic medical settings. However, this number is really an over-estimate of the number of pediatric researchers! Most subspecialists who function in academic medical centers or 


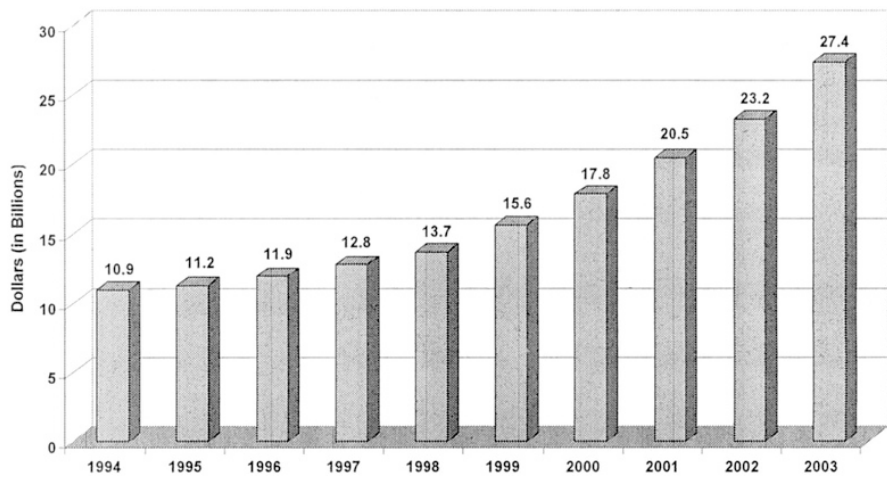

Figure 1. National Institutes of Health Budget Authorizations, 1994-2003, In Billions of Dollars. Years 1994-2000 are actual; years 2001-2003 are projected, reflecting Congressional intent to double the National Institutes of Health budget by 2003. Source: Public Budget Database, Office of Management and Budget, April 2001.

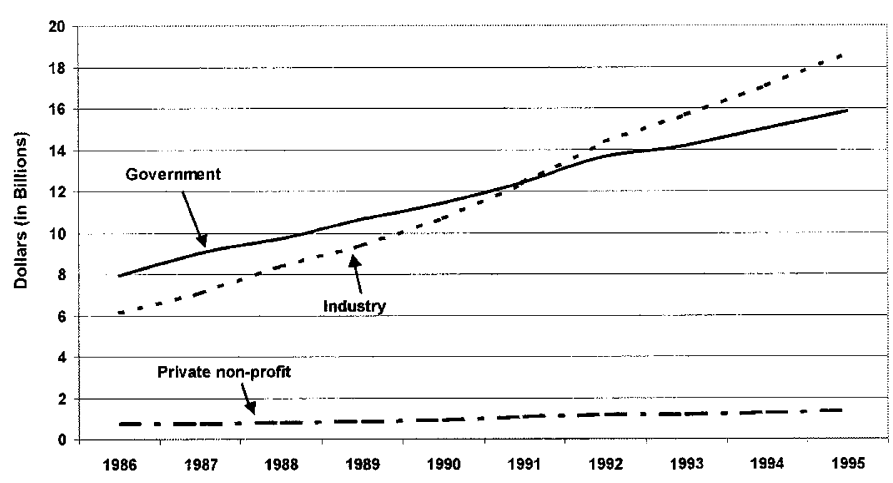

Figure 2. Estimates of National Support for Health Research and Development, in Billions of Dollars. Both government and industry support have been growing. Industry support surpassed government support in 1992. Source: Office of Extramural Research, National Institutes of Health, Estimates of National Support for Health R\&D by Source or Performer, FY 1986-1995; http:/grants.nih.gov/grants/award/trends96/pdfdocs/FEDTABLA.PDF.

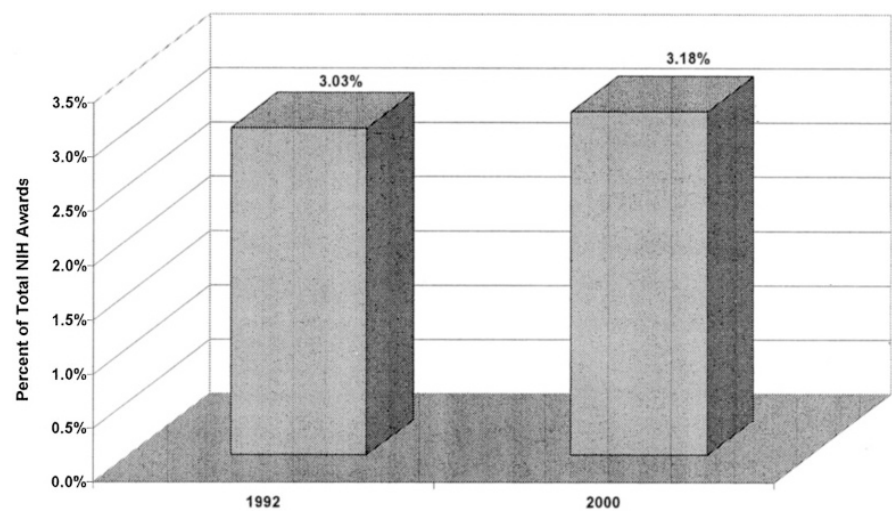

Figure 3. Pediatric Share of National Institutes of Health Research Awards in 1992 and 2000. The percentage of National Institutes of Health awards for pediatric research has changed little in the past decade. Source: Duane Alexander MD, Director, National Institute of Child Health and Human Development, National Institutes of Health.

freestanding children's hospitals primarily engage in clinical service, or in a combination of clinical service and educational activities. The majority of these subspecialists, although trained to some extent in research during their fellowships, do not actively perform substantive, cutting-edge scientific inves-

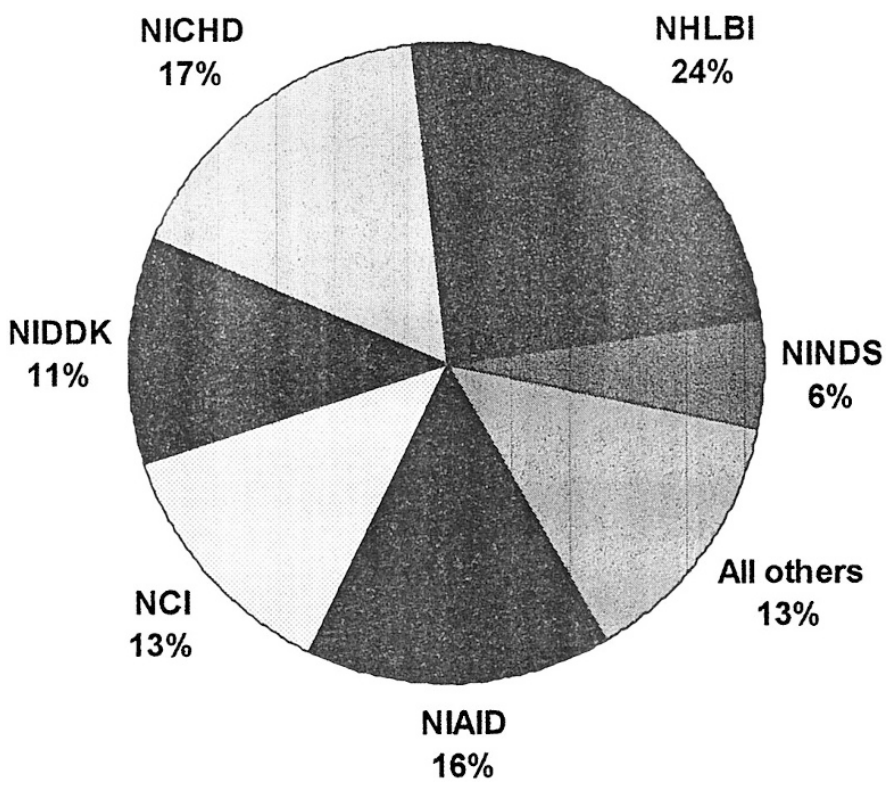

Figure 4. Sources of Pediatric Research Support by National Institutes of Health Institute. Primary sources are the National Heart, Lung, and Blood Institute (NHLBI), the National Institute of Child Health and Human Development (NICHD), the National Institute of Allergy and Infectious Diseases (NIAID), the National Cancer Institute (NCI), the National Institute of Diabetes and Digestive and Kidney Diseases (NIDDK), and the National Institute of Neurologic Disorders and Stroke (NINDS) Source: Duane Alexander MD, Director, National Institute of Child Health and Human Development, National Institutes of Health.

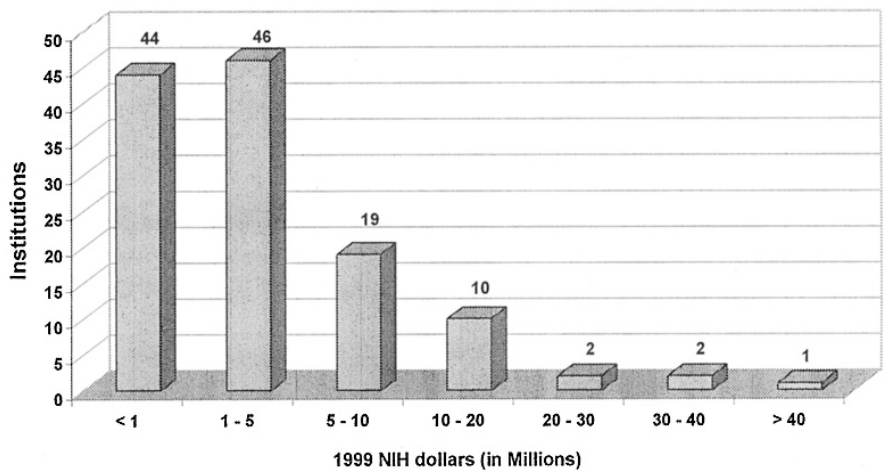

Figure 5. Total National Institutes of Health Research Funds Awarded to Pediatric Institutions. In 1999, 90 institutions received \$5 million or less in research awards from the National Institutes of Health. Five institutions received \$20 million or more in National Institutes of Health awards. Source: National Association of Children's Hospitals and Related Institutions, Children's Hospital Grants Opportunities Project, 1999.

tigation, as a primary focus of their jobs. Thus, pediatric research is performed in relatively few institutions by relatively few pediatric scientists.

What is the goal of pediatric research? Most pediatric research is focused on the singular goal of improving the lives of children by promoting health and preventing disease. The past century's successes have included advances in improved nutrition, public health measures, vaccines, antibiotics, and cancer therapy. These accomplishments have doubled our children's life expectancy and have made enormous contributions to the economy. Future pediatric research will continue to examine the environmental and psychosocial obstacles that 


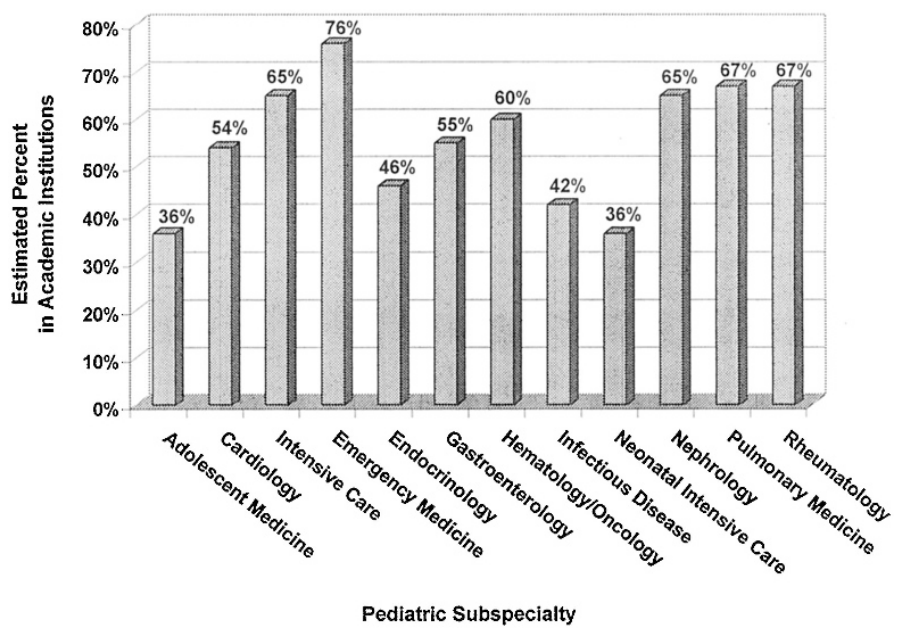

Figure 6. Estimated Percent of Pediatric Subspecialists in Academic Medicine by Subspecialty. Source: American Board of Pediatrics, 2000.

impede the ability of some children to access adequate health care. Carefully designed investigations, particularly in the arena of health services research, will play an important role in first identifying, and then eliminating, these barriers.

The dawn of the 21st century has been marked by the complete sequencing of a working draft of the human genetic code, published in February 2001. As a result of the sequencing of the human genome, a physical map of the 3 billion base pairs of nuclear DNA that constitute a human being has been constructed. Based on the information from this project, we now assume that humans have approximately 30,000 genes. By comparison, this is the same number of genes as in rodents, only slightly more than double the number of genes as in fruit flies, and only about 10,000 more than in worms ... and even plants! It is amazing to think about how similar we are to lower species - but, it is also clear the genome alone does not hold all the explanations for what it means to be human.

My prediction for pediatric research in the 21st century is that we will continue to focus on the discovery of monogenic disorders. But, there will be a far greater focus on polygenic disorders and the interactions between genes and the environment. With monogenic diseases such as sickle cell anemia or cystic fibrosis, disease is likely if the mutation is present (Fig. 8).

This is in contrast with polygenic disorders, for which there is a greater interaction between genes and the environment (Fig. 9). An individual may inherit genes that increase the susceptibility for developing a disease, for example, lung cancer. However, only after exposure to environmental factors, such as tobacco, does the disease become manifest. In the absence of inciting environmental factors, an individual with susceptibility genes might be protected from ever suffering signs or symptoms of disease (Fig. 10).

There are powerful new forces in biomedical research that will enable the discoveries of the future. These involve new research approaches or old approaches to new problems. These include the tools of genotyping, comparative genomics, proteomics, bioinformatics, pharmacogenomics, and bioethics. These new fields will be accompanied by new technologies in

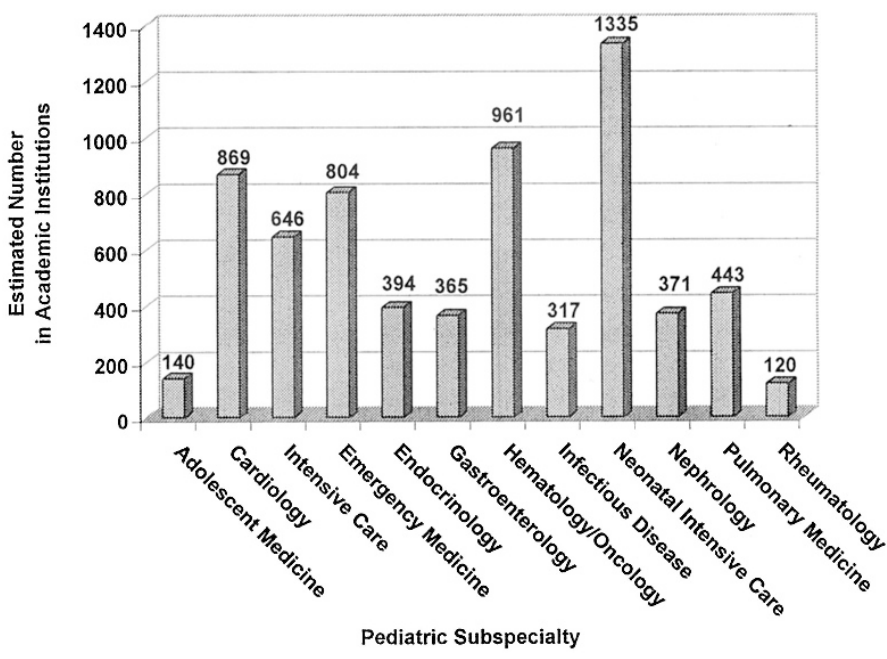

Figure 7. Estimated Number of Pediatric Subspecialists in Academic Medicine by Subspecialty. Source: American Board of Pediatrics, 2000.

\section{Monogenic Disorders}

Health

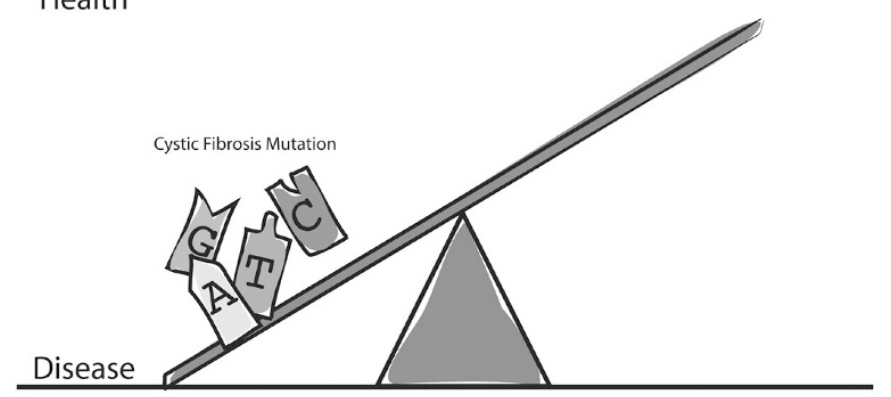

Figure 8. Monogenic Diseases. In the presence of a single gene mutation, clinical disease is likely.

\section{Polygenic Disorders}

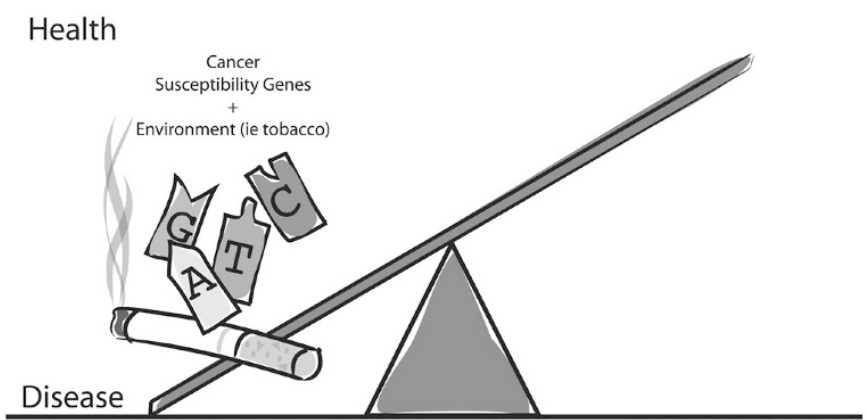

Figure 9. Polygenic Diseases. Complex diseases involve the interactions of more than one gene along with environmental factors. For example, the presence of carcinogens in tobacco smoke may increase the risk of disease in individuals genetically predisposed to cancer.

biomedical research including microchip DNA arrays, robotic sequencers, markedly more sophisticated mass spectrometry, and novel imaging systems.

Genotyping will permit us to detect genetic differences among individuals. In addition to using genotyping to determine different expression patterns among individuals, it can be 


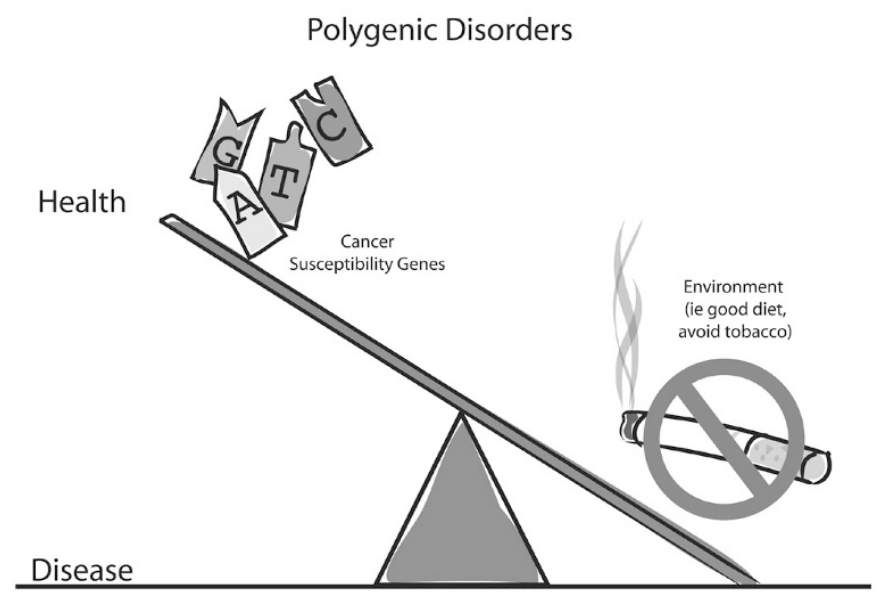

Figure 10. Polygenic Diseases. Although an individual may be genetically predisposed to a disease, the risk may be lessened with good dietary choices or avoidance of carcinogens.

used to detect differences between healthy and abnormal tissues. Thus, genotyping is an important tool for identifying the genetic factors that influence health and disease. Proteomics will be the successor to the Human Genome Project. Proteomics will permit us to determine protein structures, functions, and interactions between proteins, genes, and the environment. Proteomics is of far greater scale and complexity than the Human Genome Project. Bioinformatics uses computer models to predict gene and protein structures and can be used to identify new genes and gene products. The field of pharmacogenomics will result in new approaches to drug discovery. We will develop drugs that will be targeted to individuals. These drugs will be more effective and less toxic.

However, all of these new discoveries will lead to a flurry of complex new issues in the field of bioethics. It will become essential to deal with the protection of privacy and to tackle issues related to insurance and employment. By having so much genetic and predictive information about patients, we will need to ensure that there is good access to health care. And, we will face other pediatric bioethical issues such as the production of "designer babies."

Over the past 50 years, biomedical research has explored the molecular activities of the cell. In the past 20 years, we focused on the contents of the nucleus: the DNA, the chromosomes, and the genes. We learned about monogenic diseases and developed some very targeted drug therapies. We have taken the first steps in gene therapy and prenatal testing is now commonplace. As we move into the genomics era, we will expand our research to the more common, but more complex polygenic diseases. Armed with a new understanding of complex genomic interactions, we will focus on targeted drug therapies and preventive health care for complex diseases. New genomics technologies offer us unprecedented opportunitiesand challenges - for pediatric research in the 21 st century. In this era of genomics, proteomics, bioinformatics, pharmacogenomics, and bioethics, productive research will be dependent on a successful merger of the tools of the biologic and the digital ages. So, what is the future of pediatric research?
As stated by futurist Leonard Sweet, "The future is not something we enter ... the future is something we create."

How do we ensure that with so few pediatric investigators functioning in so few pediatric institutions, there will be productive pediatric research in the future? I have a few suggestions: Specialize, Consolidate, Innovate, Mentor and Train, and Partner (Fig. 11).

Each pediatric department and freestanding children's hospital will not be able to be expert in EVERY technologically sophisticated area important to pediatric research. Academic programs should spend time to identify their strengths and acknowledge their weaknesses. Once these are defined, then strategic planning should focus on maximizing the strengths and minimizing weaknesses by carefully selecting areas of specialization in which the particular program can excel.

Duplication of programs, technologies, personnel, and equipment is highly inefficient! Resources should be consolidated to increase economies of scale. Programs should develop areas of specialty that complement others in programs that are in close geographic proximity. Duplication should be avoided and consolidation of expensive equipment, technologies, space, and other resources should be encouraged.

The key to scientific advances is the creativity of the scientist. It is essential to provide creative scientists with an environment in which creativity will flourish. Scientists should be encouraged to apply cutting-edge technologies to pediatric problems. They should cross the classic boundaries of different disciplines to enable more creative approaches to explore pediatric research questions. Most importantly, these scientists should be allowed, and encouraged to, think outside the box!

It is essential to identify strong research candidates as early as possible and to direct them into pediatric research in medical school, or preferably, sooner. Students with a strong, passionate early desire to do research are more likely to pursue research careers. For those who have caught the research bug before residency, we should promote research opportunities during residency such as the Special Alternative Pathway and the Pediatric Research Pathway. One of the best things we can do to ensure our future is to provide training for future pediatric

\section{Pediatric Research: Suggestions for the Future}

- Specialize

- Consolidate

- Innovate

- Mentor/train

- Partner
Figure 11. Suggestions for Stimulating Further Pediatric Research. 
investigators with the best scientists around, especially those not based in pediatrics. Cross-fertilization will make us a heartier breed.

Along these lines, we should provide training in the most competitive and productive basic and clinical research environments. We should provide pediatric scientists with both pediatric and nonpediatric mentors. Importantly, we must improve the academic environment for mentors so that they can convey the passion and enthusiasm for research that once stimulated them. Finally, a good scientist does not automatically become a good mentor. We must train mentors in the art of mentorship.

The most productive research in the future will come from partnerships in science. It is important that we identify partners with complementary strengths. It does not make sense to have every laboratory or even every department or institution have expertise in every single technology just so that we can say that "we do this in our laboratory." For productive relationships to succeed, it is imperative that we develop reward and incentive programs that encourage partnerships and collaborations.

In summary, SCIMP: specialize, consolidate, innovate, mentor, train, and partner-but do not skimp on our future! The future for pediatric research in the era of genomics is bright ... if we are willing to do what the simplest bacteria do so well: evolve and adapt!

"The people who live in the past must yield to the people who live in the future. Otherwise the world would begin to turn the other way round" (Arnold Bennett, English novelist and playwright).
Acknowledgments. I would like to express my personal appreciation to Christopher Brown, Eric Schoch, and Albert William from Indiana University, Duane Alexander from NICHD, National Institutes of Health, Karen Hendricks from the Washington office of the Public Policy Council, and Walter Tunnessen from the American Board of Pediatrics; my own research mentors, Lynn Loriaux and Gordon Cutler, who together molded my career and offered me the most valuable of lessons; Dr. Richard Schreiner who has created an exceptional environment at the James Whitcomb Riley Hospital for Children, where research, clinical service, and education thrive; my friends and colleagues in the section of Pediatric Endocrinology and Diabetology; Dr. Morris Green who serves as a moral compass, advisor, and mentor; my parents who offer unconditional love and direction; my three intelligent, accomplished and modest brothers; my wonderful children, Aliza, Ari, and Naomi; and finally, my loving and supportive husband, Mark.

\section{REFERENCES}

1. Grumbach M 1990 American Pediatric Society Presidential Address at the 100th Annual Meeting: Let the Walls Come Tumbling Down. Pediatr Res 28:562-566

2. Public Budget Database, Office of Management and Budget, April 2001

3. Office of Extramural Research, National Institutes of Health, Estimates of National Support for Health R\&D by Source or Performer FY 1986-1995 http:/grants.nih.gov/ grants/award/trends96/pdfdocs/FEDTABLA.PDF

4. National Association of Children's Hospitals and Related Institutions, Children's Hospital Grants Opportunities Project, 1999

5. American Board of Pediatrics, 2000 